Conclusion All MBO patients presented with high risk of malnutrition on admission. Less than half were referred for PN. Patients who received a dietetic review and when PN was discussed at MDT were more likely to be referred for PN. It was unclear in some cases why patients were not referred for $\mathrm{PN}$. This suggest that timing of referral and integration of nutritional care, not just $\mathrm{PN}$, into clinical management discussion at MDT is crucial. Not all patients referred to the Nutrition team were discharged on HPN based on judicious review of each case.

\section{PWE-014 IS HOME PARENTERAL NUTRITION BURDENSOME IN ADVANCED CANCER PATIENTS WITH MALIGNANT BOWEL OBSTRUCTION?}

${ }^{1} \mathrm{PS}$ Patel*, 'N Keane, 'K Fragkos, 'F O'Hanlon, ${ }^{2} \mathrm{~K}$ Cauldwell, 'I Rogers, 'S Obbard, 1J Barragry, ${ }^{1} \mathrm{G}$ Sebepos-Rogers, ${ }^{1} \mathrm{~S}$ Mehta, ${ }^{1} \mathrm{~F}$ Rahman, ${ }^{1} \mathrm{~S}$ Di Caro. ${ }^{1} \mathrm{G}$ s services, University College London Hospital, London, UK; ${ }^{2}$ Palliative Care Central and North West London NHS Trust, London, UK

\subsection{6/gutjnl-2019-BSGAbstracts.345}

Introduction Use of home parenteral nutrition (HPN) in advanced cancer patients with malignant bowel obstruction (MBO) who have developed intestinal failure (IF), is controversial. Controversies relate to financial, cultural, and ethical issues, including the risk that HPN is burdensome for the patient and carer. We examined what burdens are placed when HPN is initiated.

Methods Retrospective cohort study of adults admitted to University College London Hospital with $\mathrm{MBO}$ and started on HPN between 1.1.16 and 31.12.16 with readmissions until 31.12.17. Data were analysed using mean (SD), median (range) and $\mathrm{n}(\%)$. Survival was examined using Kaplan Meier curves.

Results 10 patients with $20 \mathrm{MBO}$ admissions were started on HPN (mean \pm SD age: $55.3 \pm 13.9$ yrs, $80 \%$ female). Primary malignancies were $50 \%$ gynaecological and 50\% lower GI with metastases (70\% subdiaphragmatically). Median weight and BMI on admission were normal $(54.8 \mathrm{~kg}, 4-7 \mathrm{~kg} ; 19.1$ $\left.\mathrm{kg} / \mathrm{m}^{2}, 1-3\right)$, though, patients presented with significant weight loss $(9.1 \%, 3 .-1.1 \%)$. HPN was more likely to be set-up on the 2nd admission with BO. HPN delayed inpatient discharge by median 2 days (-) due to lack of communication within and between Oncology and Nutrition teams. Median duration of HPN was 196 days (-51). Patients were on PN 7 days/wk $(-)$, with $n=8 / 10$ utilizing nursing input for PN (dis)connection. For those who were nursed this meant -4 visits/wk by homecare nurses for PN (dis)connection. Reasons for stopping HPN completely included: death $(n=5)$, were end of life $(n=2)$ or eating (i.e. BO resolved), suggesting flexibility to stop HPN to patients' circumstances. Decision to stop HPN was not contentious in any case. Post HPN discharge, median readmissions were 3 , and 0 due to HPN. Follow-up appointments were mostly in oncology than nutrition clinics (5.5 vs 3 follow-ups). There was a $3 \mathrm{~m}$ longer survival in those on HPN (median survival: $9 \mathrm{~m}$ for HPN patients, $4 \mathrm{~m}$ in patients not on HPN). No HPN complications were observed over the 1 yr follow-up.

Conclusion HPN is more likely to be set up during the 2nd admission for MBO. HPN did not place a substantial burden on the patient with regards to readmissions and follow-up visits to hospital or discharge delays. Nursing visits, in addition to other service visits (e.g. GP, palliative care) could be perceived as burdensome by patients. These factors could potentially be alleviated by seamless integrated care among services. Survival was longer in HPN patients. Further research in larger settings and quality of life factors need to be weighed in decision making.

\section{PWE-015 THE CAUSE OF DEATH IN PATIENTS ON HOME PARENTERAL NUTRITION IN THE NORTH-EAST OF ENGLAND}

${ }^{1}$ Elisabeth Bluethner, 'Satyasheel Ramful*, 'Hayley Leyland, 'Christopher G Mountford, ${ }^{2}$ Colette L Kirk, ${ }^{2}$ Lisa Gemmell, ${ }^{1}$ Nicholas P Thompson. 'Department of Gastroenterology, Freeman Hospital, Newcastle upon Tyne, Newcastle Upon Tyne, UK; ${ }^{2}$ Department of Dietetics, Freeman Hospital, Newcastle upon Tyne, Newcastle Upon Tyne, UK

\subsection{6/gutjnl-2019-BSGAbstracts.346}

Introduction Home total parenteral nutrition (HPN) is indicated for patients with severe intestinal failure. The service at the Freeman Hospital, Newcastle upon Tyne covers a wide area encompassing Northumberland, North Cumbria, Durham, Gateshead, North Yorkshire, Tyneside, Wearside and Teesside. A previous European study. ${ }^{1}$ had suggested deaths in HPN patients after 2 years of HPN were most likely due to PN. The aim of our study was to determine whether the cause of mortality for patients who had been under our service for HPN was linked to complications of HPN therapy.

Methods This study retrospectively looked at all patients who were started on HPN by the service from 1996 until August 2018 and identified all patients who had died. The case notes were reviewed and if the cause of death was not clear from the notes, their general practitioner (GP) surgery was contacted to gain further information.

Results 88 patients were identified as having died whilst or after being on HPN. The mean age was 58 years old and their average time on HPN was 25 months. There was an even gender split of 44 in each category.

The three most common categories for HPN indication for the patients were cancer $(32 / 88,36 \%)$, inflammatory bowel disease $(13 / 88,15 \%)$ and mesenteric ischaemia $(11 / 88,13 \%)$.

Most of the deaths were attributed to cancer $(45 / 88,51 \%)$ and non-PN/non-malignant related (29/88, 33\%). There were two deaths attributed to complications of being on parenteral nutrition. For nine cases, GP surgeries did not have any further information on cause of death. We did not receive a response regarding three patients despite contacting GP surgeries

Table 1 showing cause of death according to number of years on $\mathrm{PN}$

\begin{tabular}{llll}
\multicolumn{2}{l}{ Abstract PWE-015 Table 1} & & \\
\hline & $<1$ year & - year & $>2$ years \\
\hline Cancer & 32 & 7 & 6 \\
Non-PN related & 7 & 6 & 16 \\
PN related & 0 & 0 & 2 \\
Unable to determine & 4 & 5 & 3 \\
\hline
\end{tabular}

The table above reveals that as time on HPN increased the commonest cause of mortality changes from cancer to non-PN related complications. 
On review of the patients whose cause of death were attributed to cancer, 12 did not have malignancy as initial HPN indication. Regarding mortality not linked to HPN, respiratory complications including pneumonia were the commonest causative factor $(8 / 29,28 \%)$.

Conclusions This study showed that mortality was associated with the underlying disease condition resulting in the need for HPN or other co-morbidities rather than direct complications of HPN itself.

\section{REFERENCE}

1. Pironi L, Joly F, Forbes $A$, et al. Long-term follow-up of patients on home parenteral nutrition in Europe: implications for intestinal transplantation. Gut 2011;60:1-5

\section{PWE-016 DELIVERING PARENTERAL NUTRITION- REAL WORLD CHALLENGES}

Danielle Rayner*, Sarah Harkess, Deborah Mcfarland, Sai Lee, Anjan Dhar. County Durham and Darlington Nhs Foundation Trust And Durham University, Darlington, UK

\subsection{6/gutjnl-2019-BSGAbstracts.347}

Introduction Parenteral nutrition (PN) is widely used to intravenously deliver nutrition to patients with non-functioning or inaccessible gut. The National Confidential Enquiry into Patient Outcome and Death (NCEPOD) report in 2010, "A Mixed Bag" found good practice regarding PN in only 19\% of cases. It recommended an urgent need to deliver high quality nutrition to all patients in hospital.

Aim This audit was designed to evaluate our Parenteral Nutrition Service against NCEPOD review. The primary aim was to establish whether the key recommendations of the NCEPOD report are being adhered to: documentation of indication and its appropriateness, consideration of enteral options, clinical and biochemical monitoring during $\mathrm{PN}$, duration of $\mathrm{PN}$ and any complications.

Methods All adult inpatients who received PN at Darlington Memorial Hospital and University Hospital of North Durham between June- August 2018 were identified from 2 electronic databases viz. referrals to dietetics and electronic prescribing software. Data collection was done from case notes, inpatient electronic prescription charts and pathology results. Baseline demographics, documented indication for PN, anticipated and actual duration of $\mathrm{PN}$, reason for discontinuing $\mathrm{PN}$ and associated complications were recorded and analysed using Excel ${ }^{\circledR}$. Results 49 in-patients were identified over the 3 month period, an annualised PN workload of approx. 200 pts/yr. Mean age was $69.2 \mathrm{y}(4-3)$, mean weight prior to commencing PN 75.3kg(41.-14.8kg). 22/49 pts were surgical, $1 / 49$ medical and $16 / 49$ on intensive care. $25 / 49$ pts had a clearly documented indication (ITU 5/16, medicine 10/11, surgery 10/22) but only 1 patient had a documented anticipated duration for PN. Mean duration was 10 days(-2) and mean length of inpatient stay was 21 days(-3). Only $7 / 49$ pts were reviewed by a Nutritional MDT team, although all pts were regularly reviewed by Dieticians. Reasons for discontinuing PN: oral feeding resumed in 28 pts, death/end of life care commenced in 10 pts, NG/NJ/PEG feeding commenced in 6 pts, patient refusal in 2 pts and 3 pts transferred to a tertiary service. Complications: Abnormal LFTs in 3 pts, abnormal electrolytes in 33 pts and 1 midline associated thrombophlebitis. 16/49 pts had daily testing of serum biochemistry. 30 day all-cause mortality from commencing PN was 18\% (9/49).
Conclusion Our study shows the need for a quality improvement program for PN including documentation of indications and goals of PN treatment. Patients commencing PN should be reviewed by a Nutrition MDT to improve initial assessment and monitoring. A decreased compliance with daily serum biochemistry correlates with an increased incidence of electrolyte abnormality.

Character with spaces 2999

\section{PWE-017 A MULTI-DISCIPLINARY APPROACH TO PERCUTANEOUS ENDOSCOPIC GASTROSTOMY INSERTION IN MOTOR NEURONE DISEASE}

${ }^{1} A$ Reddy*, ${ }^{2} S$ Anderson, ${ }^{3} L$ Arrandale, ${ }^{2}$ J Dunn, ${ }^{1} \mathrm{P}$ Marino, ${ }^{1} \mathrm{~PB}$ Murphy, ${ }^{1} \mathrm{~J}$ Steier, ${ }^{1} \mathrm{ES}$ Suh, ${ }^{3} \mathrm{H}$ Thomas, ${ }^{4} \mathrm{~L}$ Yin, ${ }^{1} \mathrm{M}$ Ramsay, ${ }^{1} \mathrm{~N}$ Hart, ${ }^{1} \mathrm{G}$ Kaltsakas. 'Lane Fox Respiratory Service, Guys and St Thomas' NHS Foundation Trust, London, UK; '² Department of Gastroenterology, Guys and St Thomas' NHS Foundation Trust, London, UK; ${ }^{3}$ Department of Anaesthesia, Guys and St Thomas' NHS Foundation Trust, London, UK; ${ }^{4}$ Kings College London, London, UK

\subsection{6/gutjnl-2019-BSGAbstracts.348}

Weight loss and bulbar dysfunction is common in motor neurone disease (MND). Gastrostomy placement is complex in this cohort. Percutaneous endoscopic gastrostomy (PEG) is reported as the favoured approach in patients without chronic respiratory failure with radiologically inserted gastrostomy insertion reserved for patients with severe respiratory compromise. The aim of this study was to evaluate the outcome for patients with MND receiving PEG insertion as part of a multidisciplinary team approach in a respiratory intensive care unit setting.

A retrospective observational study was conducted on MND patients on non-invasive ventilation (NIV) who were admitted for PEG insertion between September 2014 and April 2018. PEG were inserted with intravenous anaesthesia and analgesia (propofol infusion and fentanyl blouses) plus local anaesthesia to skin (lidocaine 1\%) using non-invasive mechanical ventilation on pressure-control mode.

Fifty-nine PEG insertions were performed successfully in MND patients requiring NIV and all patients discharged from hospital. Subjects (37 males) had age 65 11 years. Six (10\%)

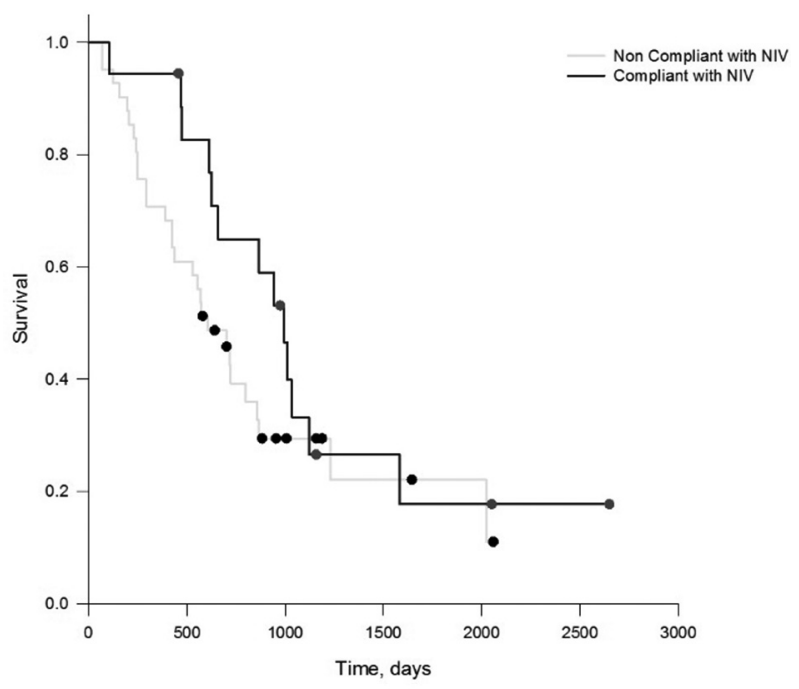

Abstract PWE-017 Figure 1 Survival of MND patients who had a PEG insertion in terms of compliance to non-invasive ventilation. 